\title{
El futuro del gas natural al 2030 como fuente energética para el desarrollo sostenible de Lima Metropolitana
}

Roger Orlando LuJan Ruiz

RECIBIDO: 11/01/2017 ACEPTADO: 18/05/2017

\begin{abstract}
RESUMEN
El presente artículo aborda el siguiente problema ¿Cómo formular un escenario al año 2030 que sea realista y a la vez sea la base para una visión factible de cumplirse para el abastecimiento de gas natural al 2030?. Para la obtención de los resultados se aplicaron encuestas así como el método de la Prospectiva Estratégica, la cual incluye los métodos MICMAC, MACTOR y SMIC. LOS principales resultados obtenidos fueron: las variables del eje estratégico, rol de actores y el escenario al 2030.
\end{abstract}

Palabras Clave: Prospectiva; Gas natural; actores; escenarios; variables.

THE FUTURE OF NATURAL GAS BY 2030 AS AN ENERGY SOURCE FOR THE SUSTAINABLE DEVELOPMENT OF LIMA METROPOLITAN

\section{ABSTRACT}

This article addresses the following problem How to formulate a scenario by the year 2030 that is realistic and at the same time is the basis for a feasible vision to be fulfilled for the supply of natural gas by 2030. To obtain the results, surveys were applied as well The Strategic Foresight method, which includes the MIC MAC, MACTOR and SMIC methods. The main results obtained were the variables of the strategic axis, role of actors, the scenario bet to 2030

Keywords: Foresight; Natural gas; actors; stages; variables.

\section{INTRODUCCIÓN}

La prospectiva, es un conjunto de análisis y estudios con el fin de explorar o de predecir el futuro. Para Fuentes (2012), la finalidad de la prospectiva es indagar en el futuro, explorar lo desconocido, imaginar lo inimaginable, procurando confeccionar modelos probables y deseables. Entre los elementos más importantes para la realización de la prospectiva se encuentran los escenarios; un escenario es el simulacro en el tiempo de los mecanismos y de los procesos inherentes a un sistema. El escenario incluye a la vez la descripción coherente del sistema en un determinado momento y la del camino que lleva a su estado final.

¿Qué se debe esperar de este energético y sus derivados? La respuesta a esto se encuentra en la forma en que se comportaría la producción mundial del petróleo, ya que la demanda a largo plazo depende más del crecimiento económico que de las políticas de sustitución o de eficiencia energética.

El principal problema de las políticas seguidas por muchos países, como en el Perú, es suponer que la oferta de petróleo se adecuará a la demanda, pero no se detienen a pensar que un mercado externo con combustibles en cantidades infinitas no existe. Éste hecho lleva a pensar en medidas con resultados a largo plazo y ordenadas en forma sistemática y progresiva que terminen en un escenario que permita un desarrollo sostenible del gas natural en Lima Metropolitana.

Los expertos consultados son:

Ing. Jorge Iván Monterroza Bula - Gerente de Operaciones - Cálidda Gas Natural del Perú

Ing. Edwin Alex Vega - Sub Gerente de Proyectos - Cálidda Gas Natural del Perú

Ing. Jorge Luis Sucari - Sub Gerente de área de Sistema SCADA - Cálidda GN del Perú

Ing. Julio Muñoz Franco - Especialista Técnico en Energía Fondo de Inclusión Social Energética

FISE - Organismo Superior de la Inversión en Energía y Minería OSINERGMIN

1 Ingeniero Químico, Consultor externo,rogger159@yahoo.com 


\section{Problema General}

¿Cuál es el futuro al 2030 del gas natural como fuente energética para el desarrollo sostenible de Lima Metropolitana?

\section{MICMAC}

Para Gabiña (1998) el método MICMAC permite establecer las relaciones indirectas y los efectos feedback entre las variables. Las 50 variables iden- tificadas por los expertos y que se han seleccionado para este trabajo corresponde a las siguientes categorías: Económico, Técnico, Operativo, Ecológico y ambiental, Político, Social y Tecnológicos. La relación de las 50 variables se puede apreciar en la Tabla 1.

Al tener 50 variables, se clasifica en su accionar y la relación existente entre cada una de ellas, motivo por el cual se procede a elaborar la matriz de

Tabla 1: Identificación de variables

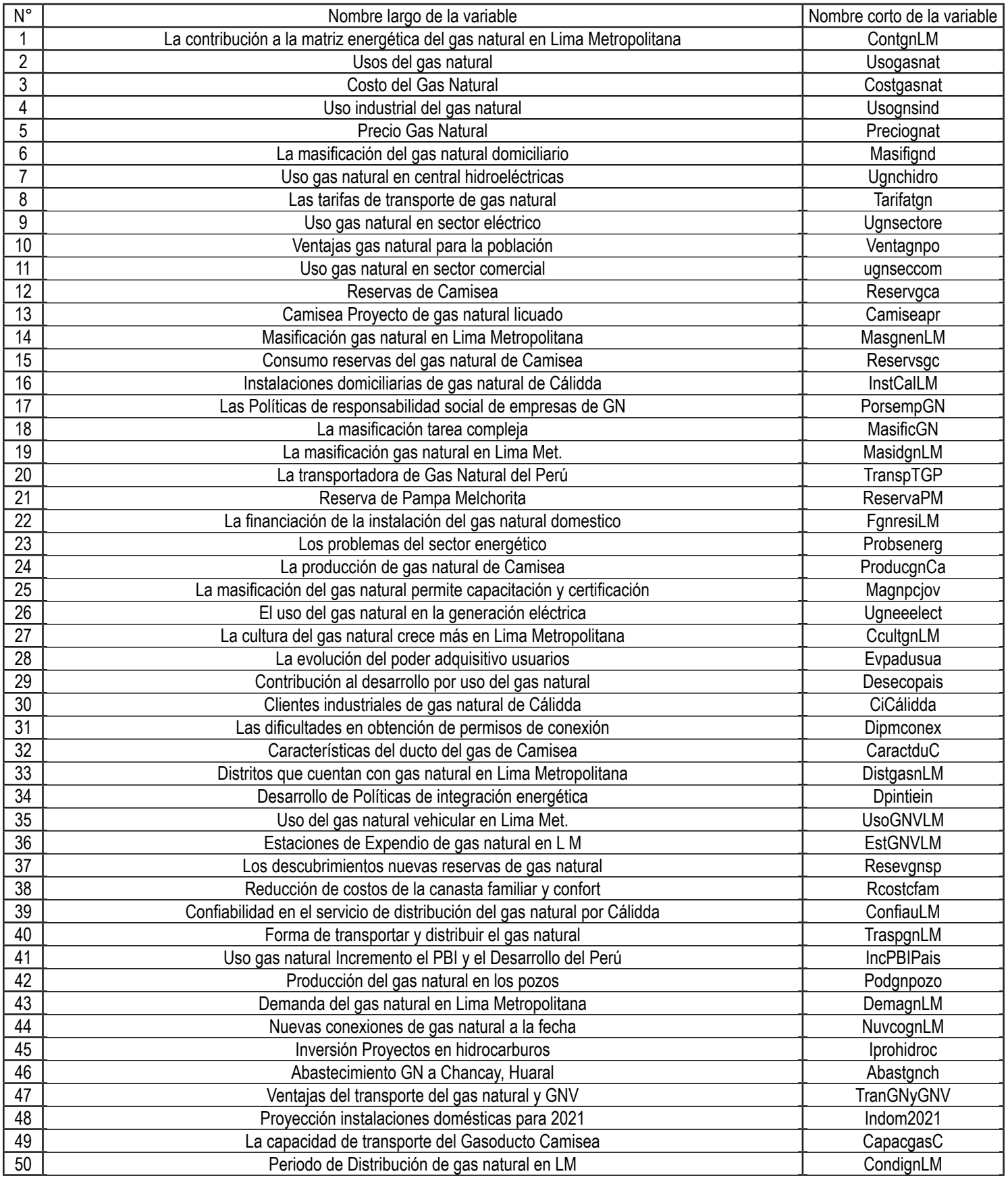

Fuente: Elaboración propia 


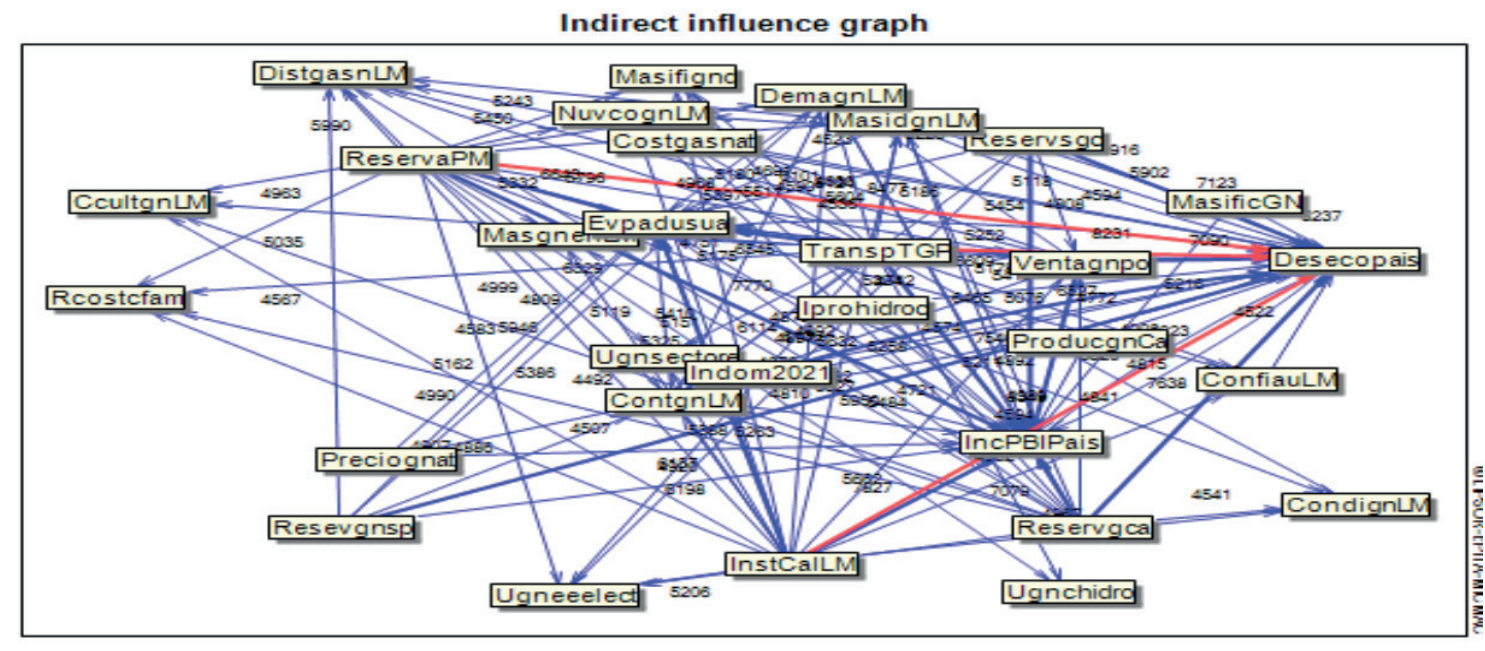

Weakest influences

Weak influences

Moderate influences

Relatively strong influences

Figura 1: Gráfico de influencias indirectas

Fuente: Elaboración propia

impactos directos MID que consiste en una matriz de doble entrada en donde se cruzan las variables para hallar su influencia y dependencia de una variable con la otra. El software que permite desplegar la figura1 es el MICMAC:

En la figura 1 las 50 variables se clasifican en: Variables Autónomas: Son las que tienen poca influencia y poca dependencia en el sistema. La gran cantidad de variables (18) pertenecen a esta categoría y son:

- CaractduC, TraspgnLM, Dipmconex, TranGNyGNV, Camiseapr, CapacgasC, Podgnpozo, PorsempGN, Probsenerg, Tarifatgr, FgnresiLM, UsoGNVLM, EstGNVLM, Dpintieir, Ugnseccom, Magnpcjov, Abastgnch, Usognsing

- Variables Resultado: Son las que tienen una alta dependencia pero una baja influencia. Las variables (5) que pertenecen a esta categoría son:

- MasgnenLM, Masifignc, Ugneeelect, Rcostcfam, DistgasnLM

- Variables Determinantes: Son las variables (11) que tienen una fuerte influencia pero una baja dependencia en el sistema. Pertenecen a esta categoría:

- TransptGP, ReservaPM, Reservgca, MasificGN, Reservsgc, ProducgnCa, Preciognnat, Iprohidroc, InstCalLM, Resevgnsp, Indom2021.

- Variables Claves: Son las variables más importantes para el sistema, son las que más influyen pero también son las más dependientes. Las variables que pertenecen a esta categoría son:
- Ventagnpo, MasidgnLM, DemagnLM, Contg$\mathrm{nLM}$, Evpadusua, Desecopais, IncPBIPais

- Variables de entorno y reguladoras: Son las variables que se encuentran cerca de la frontera y que no se pueden afirmar a que categoría pertenecen dentro del sistema. Las variables (9) que pertenecen a esta categoría son:

- CICalidda, Usogasnat, CcultgnLM, CondignLM, Ugnsectores, NUvcognLM, ConfiauLM

Podemos observar una tendencia de las variables claves, por lo que su nivel de dependencia e influencia son altos, logrando así que cree un comportamiento incierto. Haciendo que el sistema sea inestable por los cambios repentinos y/o lineales ocasionando efectos a "El futuro al 2030 del gas natural como fuente energética para el desarrollo sostenible de Lima Metropolitana".

Se debe poner mayor atención en las variables claves, ya que marcan las diferencias y los distintos futuros posibles para ese sistema.

\section{MACTOR}

MACTOR (Matriz de alianzas, conflictos: tácticas, objetivos y recomendaciones) Godet (1994), busca valorar las relaciones de fuerza entre actores y estudiar sus convergencias y divergencias con respecto a un cierto número de posturas y de objetivos asociados, asimismo, busca analizar a los actores que tienen cierto poder de mando sobre las variables. Ortegón 2006) 
Con MACTOR se busca determinar tres características de la naturaleza de los actores: relación de fuerzas entre actores, posición de los mismos y poder de mando de éstos sobre las variables.

Las variables más importantes que se han podido determinar con ayuda del MICMAC se ven influidos por diversos actores, los cuales se pretende validar e identificar de acuerdo con el método MACTOR.

Para fines prácticos se detallarán los pasos de la herramienta MACTOR en este punto; sin embargo, los cálculos en su mayoría se realizaron con la ayuda del software de LIPSOR. Para este análisis se identificaron junto con los expertos, los principales actores del sistema del estudio cuyos resultados se presentan en la tabla 2.

Tabla 2: Lista de actores identificados

\begin{tabular}{|c|l|l|}
\hline N $^{\circ}$ & Título largo & Título corto \\
\hline 1 & Proyecto Camisea & Gdcamisea \\
\hline 2 & Transportadora de Gas Natural & TGP \\
\hline 3 & Cálidda Gas Natural del Perú & CáliddaGNP \\
\hline 4 & Ministerio de Energía y Minas & Minem \\
\hline 5 & $\begin{array}{l}\text { Organismo Supervisor de la Inversión } \\
\text { en Energía y Minería }\end{array}$ & OSINERGMIN \\
\hline 6 & Gobierno Local & Municipios \\
\hline 7 & $\begin{array}{l}\text { Usuarios de Gas Natural en Lima } \\
\text { Metropolitana }\end{array}$ & UgnLM \\
\hline
\end{tabular}

Fuente: Elaboración propia

En el análisis prospectivo de "El futuro al 2030 del gas natural como fuente energética para el desarrollo sostenible de Lima Metropolitana" intervienen los siguientes "actores":

Se identificaron los objetivos propios del estudio cuyos resultados se pueden apreciar en la tabla 3.

Tabla 3: Lista de objetivos identificados

\begin{tabular}{|c|l|l|}
\hline $\mathbf{N}^{\circ}$ & Título largo & Título corto \\
\hline 1 & Modelos sustentables de Reservas & Modsusres \\
\hline 2 & Reservas probadas de gas natural & Resprobgn \\
\hline 3 & $\begin{array}{l}\text { Desarrollar un sistema de transporte de } \\
\text { gas natural }\end{array}$ & Dsistgnlm \\
\hline 4 & $\begin{array}{l}\text { Calidad y productividad del servicio de } \\
\text { transporte gas natural producido Cami- } \\
\text { sea. }\end{array}$ & Caprosegn \\
\hline 5 & $\begin{array}{l}\text { Crecimiento de la demanda de gas na- } \\
\text { tural }\end{array}$ & Crdemgnlm \\
\hline 6 & Incrementar la red de distribución & Inredgnlm \\
\hline
\end{tabular}

\begin{tabular}{|c|l|l|}
\hline 7 & $\begin{array}{l}\text { Establecer el marco legal normativo para } \\
\text { la distribución del gas natural en LM }\end{array}$ & Esmalnord \\
\hline 8 & $\begin{array}{l}\text { Promover la expansión en el uso del gas } \\
\text { natural }\end{array}$ & Proexgnlm \\
\hline 9 & $\begin{array}{l}\text { cumplimiento del marco regulatorio y las } \\
\text { normas técnicas }\end{array}$ & Cumarenot \\
\hline 10 & Regular las tarifas aplicables. & Regtaragn \\
\hline 11 & $\begin{array}{l}\text { Implementar programas y proyectos So- } \\
\text { ciales, Educativos y de Salud }\end{array}$ & Improsoce \\
\hline 12 & $\begin{array}{l}\text { Aprovechamiento de las ventajas del uso } \\
\text { del gas natural por los usuarios de Lima } \\
\text { Metropolitana }\end{array}$ & Apvegnulm \\
\hline 13 & $\begin{array}{l}\text { El tipo de usuarios del gas natural son } \\
\text { factores que determinan el consumo de } \\
\text { gas natural en Lima Metropolitana. }\end{array}$ & Ugndcgnlm \\
\hline 14 & Uso del gas natural en el sector industrial & Usgnesilm \\
\hline
\end{tabular}

Fuente: Elaboración propia

Con estos datos se procedió a aplicar la matriz de influencias directa (MID) de "actores x actores", obteniéndose el resultado en la Figura 2.

\begin{tabular}{|c|c|c|c|c|c|c|c|}
\hline MID & 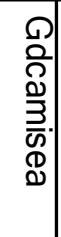 & ه & 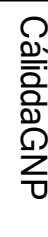 & $\frac{\nwarrow}{\frac{\mathbb{\Phi}}{3}}$ & 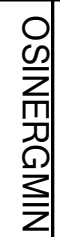 & 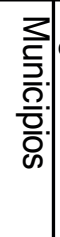 & 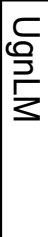 \\
\hline Gdcamisea & 0 & 3 & 2 & 0 & 0 & 0 & 3 \\
\hline TGP & 2 & 0 & 3 & 1 & 1 & 0 & 3 \\
\hline CáliddaGNP & 0 & 3 & 0 & 3 & 3 & 3 & 3 \\
\hline Minem & 3 & 3 & 3 & 0 & 1 & 0 & 1 \\
\hline OSINERGMIN & 3 & 3 & 3 & 1 & 0 & 0 & 2 \\
\hline Municipios & 0 & 0 & 2 & 0 & 0 & 0 & 2 \\
\hline UgnLM & 0 & 0 & 0 & 0 & 0 & 2 & 0 \\
\hline
\end{tabular}

Figura 2: Matriz Directa de actor por actor

Fuente: Elaboración propia

Las influencias de la matriz Directa (MDI) "Actor por actor" creado a partir de la tabla de las estrategias de los actores, Describe los actores que tienen influencia directa sobre otro.

El puntaje que se dan a las influencias son de 0 a 4 teniendo en cuenta la importancia del efecto sobre el actor:

0 : Sin influencia, 1 : Procesos, 2 : Proyectos, 3 : Misión, 4 : Existencia

Así como, en el caso MICMAC esta matriz sólo toma en cuenta las relaciones directas, por lo tanto se procedió a formular la matriz de influencias directa e indirecta (MIDI) cuyos resultados se puede apreciar en la tabla 4. 
Tabla 4: Matriz de Impacto Directo e Indirecto

\begin{tabular}{|l|c|c|c|c|c|c|c|c|}
\hline & Gdcamisea & TGP & CáliddaGNP & Minem & OSINERGMIN & Municipios & UgnLM & li \\
\hline Gdcamisea & 2 & 5 & 5 & 3 & 3 & 4 & 8 & 28 \\
\hline TGP & 4 & 7 & 7 & 5 & 5 & 5 & 10 & 36 \\
\hline CáliddaGNP & 8 & 9 & 11 & 5 & 5 & 5 & 11 & 43 \\
\hline Minem & 6 & 10 & 9 & 5 & 5 & 4 & 11 & 45 \\
\hline OSINERGMIN & 6 & 10 & 9 & 5 & 5 & 5 & 12 & 47 \\
\hline Municipios & 0 & 2 & 2 & 2 & 2 & 4 & 4 & 12 \\
\hline UgnLM & 0 & 0 & 2 & 0 & 0 & 2 & 2 & 4 \\
\hline Di & 24 & 36 & 34 & 20 & 20 & 25 & 56 & 215 \\
\hline
\end{tabular}

Fuente: Elaboración propia

De la tabla 4, se observa que el actor CaliddaGNP es el más influyente del sistema, pero al mismo tiempo uno de los más susceptibles a la influencia de los otros actores como también lo acompañan MINEM y TGP. En caso contario los municipios son los que están menos preparados para alcanzar sus objetivos y son los que están más sujetos a presión de otros.
Asimismo como en el caso de los factores, es necesario conocer el comportamiento de estos actores a fin de poder formular las estrategias para llegar a ellos y lograr su apoyo o neutralizarlos para evitar o disminuir su ataque; la figura 3 , muestra el plano de influencia y dependencia de los actores e identifica a cada actor según su posición.

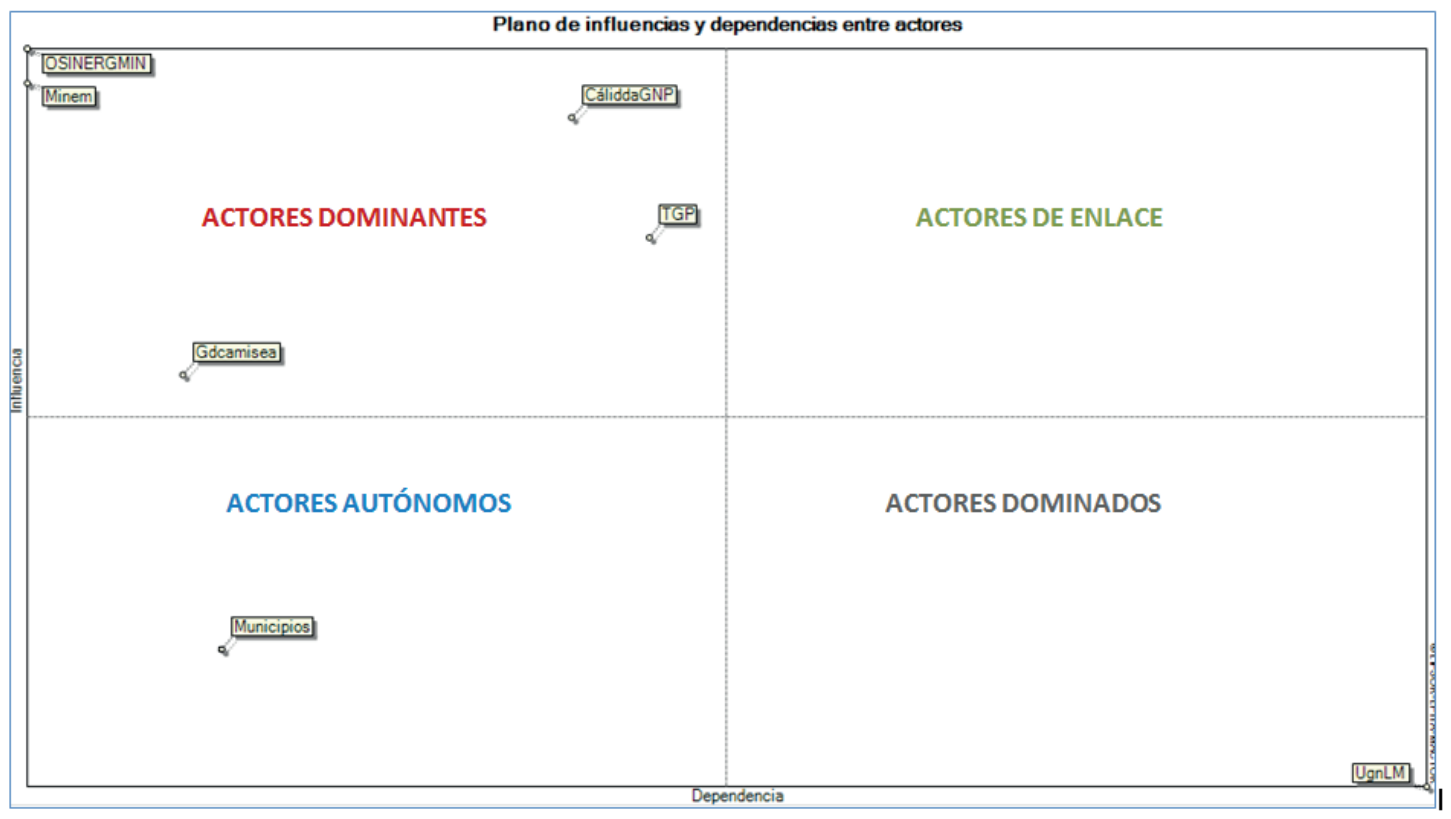

Figura 3: Influencias y dependencias entre actores

Fuente: Elaboración propia 
Los escenarios presentados a continuación, son el resultado del gráfico Histograma de extremos, sacado después de ingresar las probabilidades de ocurrencia de los 3 expertos en el sistema. La tabla 5 proporciona los escenarios posibles.

Tabla 5: Probabilidades de escenarios

\begin{tabular}{|l|c|c|c|}
\hline & Grupo 1 & Prob. $\%$ & Acum. $\%$ \\
\hline $01-11111$ & 0.348 & 34.8 & 34.8 \\
\hline $32-00000$ & 0.307 & 30.7 & 65.5 \\
\hline $05-11011$ & 0.104 & 10.4 & 75.9 \\
\hline $25-00111$ & 0.098 & 9.8 & 85.7 \\
\hline $08-11000$ & 0.028 & 2.8 & 88.5 \\
\hline $07-11001$ & 0.018 & 1.8 & 90.3 \\
\hline $21-01011$ & 0.017 & 1.7 & 92.0 \\
\hline $03-11101$ & 0.015 & 1.5 & 93.5 \\
\hline $09-10111$ & 0.012 & 1.2 & 94.7 \\
\hline $10-10110$ & 0.01 & 1 & 95.7 \\
\hline $23-01001$ & 0.01 & 1 & 96.7 \\
\hline $12-10100$ & 0.009 & 0.9 & 97.6 \\
\hline $17-01111$ & 0.008 & 0.8 & 98.4 \\
\hline $16-10000$ & 0.005 & 0.5 & 98.9 \\
\hline $29-00011$ & 0.005 & 0.5 & 99.4 \\
\hline $26-00110$ & 0.003 & 0.3 & 99.7 \\
\hline $28-00100$ & 0.002 & 0.2 & 99.9 \\
\hline $04-11100$ & 0.001 & 0.1 & 100.0 \\
\hline $02-11110$ & 0 & 0 & 100.0 \\
\hline $06-11010$ & 0 & 0 & 100.0 \\
\hline $11-10101$ & 0 & 0 & 100.0 \\
\hline $13-10011$ & 0 & 0 & 100.0 \\
\hline $14-10010$ & 0 & 0 & 100.0 \\
\hline $15-10001$ & 0 & 0 & 100.0 \\
\hline $18-01110$ & 0 & 0 & 100.0 \\
\hline $19-01101$ & 0 & 0 & 100.0 \\
\hline $20-01100$ & 0 & 0 & 100.0 \\
\hline $22-01010$ & 0 & 0 & 100.0 \\
\hline $24-01000$ & 0 & 0.0 .0 \\
\hline $27-00101$ & 0 & 0 & \\
\hline $30-00010$ & 0 & 0 & 0.0 \\
\hline $31-00001$ & 0 & 0 & 0 \\
\hline
\end{tabular}

Fuente: Elaboración propia

\section{CONCLUSIONES}

Escenario 01-11111 (Se cumplen todas las hipótesis): El Paraíso - Todos por un buen camino. Probabilidad de realización: $\mathbf{3 4 . 8 \%}$

En el año 2030 la producción de gas natural se habrá incrementado debido a las reservas probadas de gas natural en los lotes 88 y 56 . Además que el cambio de matriz energética está asegurado en el país por las reservas registradas y a las inversiones que se harán para la exploración y explotación enfocadas en la mejoras e incremento de la producción; esto, gracias a la solidez del sistema político y el prudente manejo de la economía en un prolongado periodo de prosperidad ha creado condiciones para las inversiones de manera que los recursos de inversión proviene del sector privado. De esta manera el uso del gas natural continuará contribuyendo al desarrollo económico del País. El adecuado uso de este recurso logrará dar la solución a problemas de desabastecimiento de gas natural en el país, utilizándolo como herramienta de desarrollo sustentable para todos los peruanos.

Escenario 32-00000 (No se cumplen todas las hipótesis): Hacia el precipicio - El Final - La gran pérdida. Probabilidad de realización: $30.7 \%$

En el año 2030 la producción de gas natural no se habrá incrementado debido a que no se probaron las reservas de gas natural en los lotes 88 y 56 , por lo que la matriz energética no está asegurado en el país por reservas no registradas $Y$ que no habrían inversiones para la exploración y explotación y no habría mejoras ni incremento en la producción; todo esto debido a un débil sistema político y un inestable economía que no crea condiciones para las inversiones. El Perú no logrará contar con una política energética alineada al desarrollo socioeconómico del país, donde todos sean protagonistas y destinatarios del desarrollo sustentable, empezando con los poderes del Estado, las autoridades regionales, entidades representativas, trabajadores y el resto de la sociedad.

Escenario 05-11011 (Se cumplen todas las hipótesis menos la tercera hipótesis): Un traspiés - 0 Buen inicio con una llamada de atención para seguir a un buen ritmo. Probabilidad de realización: $10.4 \%$

En el año 2030 la producción de gas natural se habrá incrementado debido a las reservas probadas de gas natural en los lotes 88 y 56 . Además que el 
cambio de matriz energética está asegurado en el país por las reservas registradas $Y$ a las inversiones que se harán para la exploración y explotación enfocadas en la mejoras e incremento de la producción; esto, gracias a la solidez del sistema político y el prudente manejo de la economía en un prolongado periodo de prosperidad ha creado condiciones para las inversiones de manera que los recursos de inversión proviene del sector privado. De esta manera el uso del gas natural continuará contribuyendo al desarrollo económico del País. El adecuado uso de este recurso logrará dar la solución a muchos problemas de nuestro país, utilizándolo como herramienta de desarrollo sustentable para todos los peruanos.

\section{REFERENCIAS BIBLIOGRÁFICAS}

[1] Fuentes, A. (2012). Visión Gerencial. Prospectiva de gestión y estrategia empresarial. Barcelona.: Editorial Lulu

[2] Gabiña, J. (1998). Prospectiva y ordenación del territorio: hacia un proyecto de futuro. Barcelona.: Editorial Marcombo

[3] Godet, M. (1994). From Anticipation to Action. Paris: Unesco Publishing

[4] Ortegon, E. (2006). Manual de prospectiva y decisión estratégica: bases teóricas e instrumentos. Santiago de Chile: Ed. Naciones Unidas. 